\title{
Trombosis venosa portal posesplenectomía por anemia hemolítica. Reporte de caso y revisión de la literatura
}

\section{Case Report of Venous Portal Vein Thrombosis after Splenectomy to Treat Hemolytic Anemia and Review of the Literature}

\author{
William A. Sarmiento-Burbano, ${ }^{1}$ William Otero-Regino, ${ }^{2 \star}$ Julián Parga Bermúdez. ${ }^{3}$
}

\footnotetext{
1 Residente de medicina interna, Universidad Naciona de Colombia. Bogotá D. C., Colombia.

2 Profesor titular de medicina, coordinador de gastroenterología, Universidad Nacional de Colombia, Hospital Universitario Nacional.

Gastroenterólogo, Clínica Fundadores. Bogotá D. C. Colombia,

3 Internista, fellow de gastroenterología, Universidad Nacional de Colombia, Hospital Universitario Nacional. Bogotá D. C., Colombia.

*Correspondencia: waoteror@gmail.com.
}

Fecha recibido: $\quad 14 / 02 / 18$ Fecha aceptado: 10/01/19

\begin{abstract}
Resumen
La trombosis venosa portal secundaria a esplenectomía es una patología frecuente. En pacientes llevados a este procedimiento por anemia hemolítica, varios factores de riesgo locales y sistémicos contribuyen a su presentación. Esta complicación es potencialmente mortal y ha existido gran discusión sobre las intervenciones adecuadas para su prevención y tratamiento. Frecuentemente se desconoce esta alteración. Se presenta un caso y se hace una revisión del tema.
\end{abstract}

\section{Palabras clave}

Esplenectomía, trombosis venosa portal, anemia hemolítica, várices gástricas.

\begin{abstract}
Portal vein thrombosis is a frequently occurring pathology following splenectomy. Several local and systemic risk factors contribute to its occurrence among patients who undergo this procedure to treat hemolytic anemia. This potentially fatal complication has caused great discussion about appropriate interventions for its prevention and treatment. Frequently this alteration is undetected. We present a case and review the topic.
\end{abstract}

Keywords

Splenectomy, portal venous thrombosis, hemolytic anemia, gastric varices.

\section{INTRODUCCIÓN}

La trombosis venosa portal (TP) secundaria a esplenectomía es una importante patología, frecuentemente olvidada y potencialmente mortal (1). Su incidencia varía del $5 \%$ al $50 \%$ (2). La mortalidad se reduce de $5 \%$ a $0 \%$ si se diagnostica e interviene tempranamente $(3,4)$. Con frecuencia, clínicos y cirujanos no tienen en cuenta esta potencial complicación, por lo que el paciente no recibe profilaxis y consulta cuando ya tiene instaurada la hipertensión portal con sus complicaciones, y en adelante debe ser controlado permanentemente con hospitalizaciones, transfusiones y demás. Se presenta el caso de una paciente con TP secundaria a esplenectomía.

\section{CASO CLÍNICO}

Mujer colombiana de 37 años de edad, docente de bachillerato, vive en unión libre. A los 12 años se le diagnosticó esferocitosis hereditaria y 6 años después se le hizo esplenectomía. A los 2 años posesplenectomía consultó por hematemesis y melenas. En la endoscopia le encontraron várices esofágicas sangrantes, las cuales se trataron con escleroterapia y se inició tratamiento con propranolol. El eco Doppler portal mostró "trombo hipoecogénico en la porta y su rama derecha con importante circulación colateral e hipertensión portal". La resonancia magnética (RM) demostró degeneración cavernomatosa de la vena porta. Ha tenido múltiples ingresos por sangrado digestivo 
superior secundario a gastropatía por hipertensión portal y ha recibido tratamiento del episodio agudo con soporte hidroelectrolítico, terlipresina y transfusiones de glóbulos rojos; ambulatoriamente, propranolol.

Hace 3 años se le encontraron várices gástricas sin sangrado. En los últimos 2 años se le encontró elevación de aminotransferasas 3 veces por encima del límite superior normal; bilirrubina total: $1,5 \mathrm{mg} \%$ y directa: $0,8 \mathrm{mg} \%$; albúmina: 3,4 g \%; hipergamaglobulinemia; y tuvo una biopsia hepática sin fibrosis.

En octubre de 2017 tuvo hematemesis; la endoscopia alta mostró várices cardiales y subcardiales, con signos de reciente sangrado, las cuales se ligaron sin complicaciones (Video 1). También se encontraron várices gástricas, no sangrantes. No ha vuelto a sangrar, recibe tratamiento con propranolol y es controlada por gastroenterología. Se tiene previsto el tratamiento de las várices gástricas con cianoacrilato y lipiodol si llegan a sangrar.

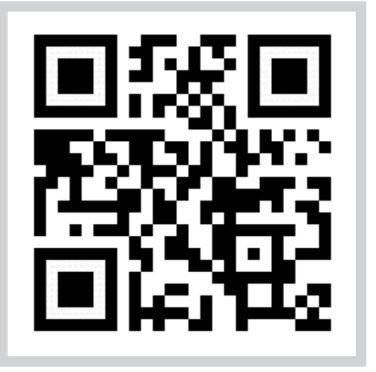

Escanee el código con su smartphone

Video 1. Ligadura de várices subcardiales por hipertensión portal poseplenectomía.

\section{METODOLOGÍA}

Para la presente revisión se hizo una búsqueda en Medline a través de la interfase Pubmed con los siguientes términos: ("portal vein" [MeSH Terms] OR ("portal" [All Fields] AND "vein" [All Fields]) OR "portal vein" [All Fields]) AND ("thrombosis" [MeSH Terms] OR "thrombosis" [All Fields]) AND ("splenectomy” [MeSH Terms] OR "splenectomy" [All Fields]), con 478 resultados. Los autores seleccionaron 53 artículos. De esos, 42 son de los últimos 10 años y están relacionados con fisiopatología, historia natural, diagnóstico, complicaciones agudas y crónicas, tratamiento de la TP posesplenectomía y tratamiento de várices esofágicas y gástricas. Se identificaron 30 estudios primarios, 6 metaanálisis y revisiones sistemáticas, 15 revisiones narrativas y 2 guías de práctica clínica. Se incluyeron 10 artículos anteriores a la última década por su aporte histórico al entendimiento de esta patología.

\section{REVISIÓN DEL TEMA}

La esplenectomía total es el tratamiento de elección para los pacientes con anemia hemolítica severa secundaria a esferocitosis, la cual disminuye o elimina la anemia $(5,6)$. Sin embargo, esta cirugía puede producir trombosis de la vena porta (TVP) (5).

\section{DEFINICIONES}

La TVP y el síndrome de Budd-Chiari forman parte de la trombosis venosa esplácnica $(6,7)$. Por su conformación anatómica, la TVP puede estar en las ramas portales intrahepáticas, la porta extrahepática, la vena mesentérica superior y la vena esplénica (5-7). El síndrome de obstrucción venosa portal de origen extrahepático (OVPEH) involucra estas regiones y, por definición, excluye la TP relacionada con cirrosis y hepatocarcinoma, que tienen fisiopatología y tratamiento (6).

\section{FISIOPATOLOGÍA}

En la actualidad aún se desconocen los mecanismos responsables de la TVP posesplenectomía. Sin embargo, se considera que están relacionados con la tríada clásica de Virchow: estasis venosa, hipercoagulabilidad y lesión endotelial (7). Diversos trabajos han demostrado que la resección del bazo disminuye el flujo sanguíneo portal y produce la turbulencia y estasis en el muñón, los cuales aumentan los diámetros de la porta y de la vena esplénica $(7,8)$; está condición predispone a trombosis $(8)$. Cuando el diámetro preoperatorio de la vena esplénica es $>8 \mathrm{~mm}$, el riesgo de TP es 2,57 (intervalo de confianza [IC] $95 \%$ : $1,26-5,23)(9)$ y si el de la vena porta es $>13 \mathrm{~mm}$, el riesgo aumenta 5,7 veces (IC 95 \%: 2,68-12,09) (8). Asimismo, la esplenomegalia y el peso esplénico $>1 \mathrm{~kg}$ aumentan el riesgo de TVP 14 veces (10).

También se ha considerado que la cirugía laparoscópica puede asociarse con estasis sanguínea e hipercoagulabilidad de la circulación portal, relacionados con el neumoperitoneo con dióxido de carbono $\left(\mathrm{CO}_{2}\right)$ y la posición antitrendelenburg $(11,12)$. La reducción del flujo sanguíneo portal y hepático produce daño en las células del endotelio vascular y promueve la exposición de componentes tisulares capaces de desencadenar coagulación $(13,14)$. Se ha encontrado que desde el tercer día postoperatorio aumentan las plaquetas y los niveles del inhibidor de plasminógeno tisular tipo 1, activadores del plasminógeno y del tiempo de tromboplastina parcial activada, lo que favorece la TVP (15). 


\section{MANIFESTACIONES CLÍNICAS}

La TVP puede aparecer desde el postoperatorio inmediato hasta varios meses e incluso 3 años después de la cirugía (16). En general, los síntomas son leves e inespecíficos, con dolor abdominal o fiebre, aunque el proceso puede ser asintomático (17). Por lo anterior, el diagnóstico temprano es inusual; es más frecuente que la TVP se diagnostique cuando ya hay complicaciones como sangrado varicoso (17-19). Ocasionalmente, el sangrado puede ocurrir décadas después de la esplenectomía y la TVP (18).

\section{COMPLICACIONES AGUDAS DE LA TVP}

La TVP se puede acompañar de trombosis de la vena mesentérica, con riesgo de infarto intestinal en un tercio de los pacientes, con riesgo de mortalidad en el $20 \%$ (19). Esta complicación asociada se presenta agudamente con dolor abdominal y ocasionalmente con diarrea, náuseas, vómito y sangrado digestivo bajo (19).

\section{DIAGNÓSTICO}

El método clásicamente utilizado para el diagnóstico de TVP es el Doppler portal, cuya sensibilidad es de $60 \%$ al $100 \%$ dependiendo del operador (20). La tomografía axial computarizada (TAC) y la RM pueden ayudar a determinar la extensión de la trombosis $(21,22)$. Estas 2 últimas son útiles cuando el ultrasonido es difícil por la interposición de gas que impide identificar la trombosis mesentérica (21). El hemograma puede mostrar leucocitosis y trombocitosis (22). Con frecuencia hay elevación de la proteína $C$ reactiva (PCR) en los primeros 7 días después de la cirugía, independientemente de los síntomas (18). El perfil hepático puede mostrar colestasis y elevación de amilasa 1,5 veces por encima del límite normal, aunque generalmente los laboratorios pueden ser normales (3).

\section{PROFILAXIS}

Por la relativa alta frecuencia, se ha recomendado profilaxis con heparina de bajo peso molecular desde las primeras 12 a 24 horas después del procedimiento y continuarla por lo menos durante 4 semanas (23-25), y más aún cuando están presentes los factores de riesgo preoperatorios mencionados (26).

\section{TRATAMIENTO DE LA TP ESTABLECIDA}

La piedra angular del tratamiento es la anticoagulación (27, 28). Se utiliza heparina de bajo peso molecular y simultáneamente warfarina hasta obtener un índice internacional normalizado (INR) entre 2-3, y después de lograr esta meta del INR, se debe continuar con warfarina durante 3 a 6 meses (27). Hasta el momento, no hay estudios sobre el uso de inhibidores directos de la trombina, como el dabigatrán o del factor Xa, como el rivaroxabán y apixabán (28).

La trombólisis se recomienda para tratar la TVP aguda, especialmente cuando la trombosis es extensa y hay también compromiso de la vena mesentérica superior $(28,29)$. Esta puede administrarse localmente o sistémica con tasas de éxito variables (29). La administración local puede producir sangrado mayor hasta en el $50 \%$ de los pacientes (29). No obstante, la infusión transcatéter de urocinasa en la arteria mesentérica superior parece ser más segura (30). La trombólisis dirigida por catéter vía transyugular intrahepática es otra opción de tratamiento, aunque se requiere experiencia con este procedimiento (31). La administración sistémica de trombolíticos tiene menos riesgo de sangrado siguiendo estrictamente los criterios de exclusión (32).

\section{ALTERNATIVAS DE TRATAMIENTO DE LA TVP}

El shunt o derivación portosistémica vía transyugular intrahepática (TIPS) permite la recanalización directa con catéter o balón y la administración de trombolíticos (32). Este procedimiento se debe hacer en centros con alta experiencia para disminuir la disfunción de la derivación y retrombosis (33). La angioplastia percutánea con balón o colocación de stent vía transhepática o esplénica es otro procedimiento útil, aunque sus tasas de retrombosis son de $9 \%-40 \%(34)$.

\section{COMPLICACIONES CRÓNICAS DE LA TP}

En la TVP crónica puede producirse OVPEH, en la que la vena porta se reemplaza por tejido fibroso y se desarrollan colaterales periportales formando un cavernoma (35). Este síndrome en algunos casos puede asociarse con hipertensión portal, gastropatía portal, várices gástricas, colangiopatía portal y encefalopatía hepática (36). Cuando existe transformación cavernomatosa de la porta, la anticoagulación no está indicada $(35,36)$. En todos los casos se deben tratar todas las complicaciones derivadas de la PVP (36).

\section{VÁRICES GÁSTRICAS}

Pueden ser esofagogástricas o gástricas aisladas, estas últimas son las más frecuentes (37). En las gástricas, las cardiofúndicas explican la mayoría de los casos $(37,38)$. Estas várices están asociadas en un $65 \%-85 \%$ con derivaciones gastrorrenales y son responsables del $5 \%-10 \%$ del sangrado digestivo alto en esos pacientes (39). 


\section{Tratamiento de las várices gástricas}

Durante los episodios de sangrado, la primera opción de tratamiento es la obliteración con cianoacrilato y la segunda es la ligadura con bandas (40). El tratamiento con inyección de cianoacrilato controla el sangrado en el $89 \%-94 \%$, con resangrado del $26 \%-31 \%$ (41). Con la ligadura, el éxito para controlar el sangrado de várices gástricas pequeñas es de $80 \%$, con resangrado del $48 \%-89 \%$ principalmente en várices gástricas IGV 1 de la clasificación de Sarin (42). Aunque ambos métodos pueden lograr el control del sangrado agudo, la tasa de resangrado es menor con cianoacrilato; y cuando las várices no son pequeñas, el tratamiento de elección es la escleroterapia con ese medicamento (43).

El ultrasonido endoscópico (USE) permite verificar la administración intraluminal de cianoacrilato, con riesgo de resangrado del $19 \%$, ofreciendo la posibilidad de detectar várices residuales (44). La ligadura aislada no es recomendable, aunque se puede complementar con inyección de cianoacrilato (45). No existen trabajos de tratamiento endoscópico profiláctico de las várices gástricas en pacientes con TVP. En pacientes con cirrosis y várices gástricas se encontró solo un estudio aleatorizado (46). En ese trabajo se menciona que los pacientes en el grupo de cianoacrilato tuvieron menos sangrado (15\%) que aquellos con bloqueantes $\beta$ no selectivos (38\%) o sin intervención (53\%). $\mathrm{Al}$ igual que en los pacientes con sagrado agudo por várices esofágicas, el tratamiento inicial incluyó resucitación con líquidos parenterales, medicamentos vasoactivos y antibióticos antes del tratamiento endoscópico (37).

Otra terapia emergente es la inyección de trombina, la cual es exitosa en $70 \%-100 \%$, con una tasa de resangrado del $0 \%-27 \%$ (47). Cuando hay compromiso hemodinámico severo, la terapia puente de taponamiento con balón durante 24-48 horas es una importante alternativa, con la cual se logra detener el sangrado en el $80 \%$ de los casos. La obliteración transvenosa retrógrada con balón de oclusión (BRTO) es una intervención que aprovecha la derivación gastrorrenal para obliterar de forma retrógrada las várices gástricas (48). En casos refractarios se puede recurrir a los TIPS (49). La utilización de vasoactivos se basa en la eficacia para controlar el sangrado de las várices esofágicas; por ende, se necesitan estudios epidemiologicos en pacientes con sangrado por várices gástricas (49).

\section{CIRROSIS POR TP}

En algunos casos, la TVP puede producir cirrosis (50). Los mecanismos involucrados pueden incluir isquemia, la cual induciría apoptosis, atrofia y regeneración nodular, con eventual fibrosis y distorsión histológica que, dependiendo de su magnitud, finalmente pueden llevar a cirrosis en un hígado previamente sano (51).

\section{CONCLUSIONES}

Los pacientes esplenectomizados por anemia hemolítica tienen un alto riesgo de TP y, por tanto, está indicada la profilaxis durante 1 mes después del procedimiento. El tratamiento de esta entidad requiere anticoagulación mínimo por 6 meses. La trombosis mesentérica e infarto intestinal o la hipertensión portal con sangrado por várices esofagogástricas son complicaciones relacionadas con alta mortalidad. El sangrado agudo por várices esofágicas o gástricas se hace similar a los pacientes con cirrosis e hipertensión portal (52).

\section{Conflictos de interés}

Ninguno.

\section{REFERENCIAS}

1. Targarona EM. Portal vein thrombosis after laparoscopic splenectomy: the size of the risk. Surg Innov. 2008;15(4):266-70. doi: 10.1177/1553350608324931.

2. Gelas T, Scalabre A, Hameury F, Dubois R, Grosos C, Mouriquand PD, et al. Portal vein thrombosis after laparoscopic splenectomy during childhood. J Thromb Thrombolysis. 2014;38(2):218-22. doi: 10.1007/s11239013-1037-2.

3. Vecchio R, Cacciola E, Cacciola RR, Marchese S, Intagliata E. Portal vein thrombosis after laparoscopic and open splenectomy. J Laparoendosc Adv Surg Tech A. 2011;21(1):715. doi: 10.1089/lap.2010.0325.

4. Stamou KM, Toutouzas KG, Kekis PB, Nakos S, Gafou A, Manouras A, et al. Prospective study of the incidence and risk factors of postsplenectomy thrombosis of the portal, mesenteric, and splenic veins. Arch Surg. 2006;141(7):663-9.doi: 10.1001/archsurg.141.7.663.

5. Rice HE, Englum BR, Rothman J, Leonard S, Reiter A, Thornburg C, et al. Clinical outcomes of splenectomy in children: report of the splenectomy in congenital hemolytic anemia registry. Am J Hematol. 2015;90(3):187-92. doi: 10.1002/ajh.23888.

6. Acosta S, Alhadad A, Svensson P, Ekberg O. Epidemiology, risk and prognostic factors in mesenteric venous thrombosis. Br J Surg. 2008;95(10):1245-51. doi: 10.1002/bjs.6319.

7. Ponziani FR, Zocco MA, Garcovich M, D’Aversa F, Roccarina D, Gasbarrini A. What we should know about portal vein thrombosis in cirrhotic patients: a changing perspective. World J Gastroenterol. 2012;18(36):5014-20. doi: 10.3748/wjg.v18.i36.5014.

8. Li MX, Zhang XF, Liu ZW, Lv Y. Risk factors and clinical characteristics of portal vein thrombosis after splenectomy 
in patients with liver cirrhosis. Hepatobiliary Pancreat Dis Int. 2013; 12(5):512-9.

9. de'Angelis N, Abdalla S, Lizzi V, Esposito F, Genova P, Roy $\mathrm{L}$, et al. Incidence and predictors of portal and splenic vein thrombosis after pure laparoscopic splenectomy. Surgery. 2017;162(6):1219-1230. doi: 10.1016/j.surg.2017.07.016.

10. Ushitora Y, Tashiro H, Takahashi S, Amano H, Oshita A, Kobayashi T, et al. Splenectomy in chronic hepatic disorders: portal vein thrombosis and improvement of liver function. Dig Surg. 2011;28(1):9-14. doi: 10.1159/000321886.

11. Wang M, Zhang M, Li J, Zhou J, Wu Z, Peng B. Risk factors of portal vein thrombosis in patients with beta thalassemia major after splenectomy: laparoscopic versus open procedure. Hepatogastroenterology. 2014;61(129):48-54.

12. Cheng J, Tao K, Yu P. Laparoscopic splenectomy is a better surgical approach for spleen-relevant disorders: a comprehensive meta-analysis based on 15-year literatures. Surg Endosc. 2016;30(10):4575-88. doi: 10.1007/s00464-0164795-z.

13. Rottenstreich A, Kleinstern G, Spectre G, Da'as N, Ziv E, Kalish Y. Thromboembolic Events Following Splenectomy: Risk Factors, Prevention, Management and Outcomes. World J Surg. 2018;42(3):675-681. doi: 10.1007/s00268017-4185-2.

14. Lee DH, Barmparas G, Fierro N, Sun BJ, Ashrafian S, Li T, et al. Splenectomy is associated with a higher risk for venous thromboembolism: A prospective cohort study. Int J Surg. 2015;24(Pt A):27-32. doi: 10.1016/j.ijsu.2015.10.011.

15. Pommerening MJ, Rahbar E, Minei K, Holcomb JB, Wade $\mathrm{CE}$, Schreiber MA, et al. Splenectomy is associated with hypercoagulable thrombelastography values and increased risk of thromboembolism. Surgery. 2015;158(3):618-26. doi: 10.1016/j.surg.2015.06.014.

16. Pietrabissa A, Moretto C, Antonelli G, Morelli L, Marciano E, Mosca F. Thrombosis in the portal venous system after elective laparoscopic splenectomy. Surg Endosc. 2004;18(7):1140-3.doi: 10.1007/s00464-003-9284-5.

17. Borrazzo EC, Daly JM, Morrisey KP, Fischer E, Belmont M, Hogle NJ, et al. Hand-assisted laparoscopic splenectomy for giant spleens. Surg Endosc. 2003;17(6):918-20. doi: 10.1007/s00464-002-8946-z.

18. Ruiz-Tovar J, Priego P. Portal Vein Thrombosis After Splenic and Pancreatic Surgery. Adv Exp Med Biol. 2017;906:241251. doi: 10.1007/5584_2016_119.

19. Condat B, Valla D. Nonmalignant portal vein thrombosis in adults. Nat Clin Pract Gastroenterol Hepatol. 2006;3(9):505-15. doi: 10.1038/ncpgasthep0577.

20. Ponziani FR, Zocco MA, Campanale C, Rinninella E, Tortora A, Di Maurizio L, et al. Portal vein thrombosis: insight into physiopathology, diagnosis, and treatment. World J Gastroenterol. 2010;16(2):143-55.doi: 10.3748/ wjg.v16.i2.143.

21. Lee HK, Park SJ, Yi BH, Yeon EK, Kim JH, Hong HS. Portal vein thrombosis: CT features. Abdom Imaging. 2008;33(1):72-9. doi: 10.1007/s00261-007-9200-x.
22. De Stefano V, Martinelli I. Splanchnic vein thrombosis: clinical presentation, risk factors and treatment. Intern Emerg Med. 2010;5(6):487-94. doi: 10.1007/s11739-010-0413-6.

23. Zhang N, Yao Y, Xue W, Wu S. Early prophylactic anticoagulation for portal vein system thrombosis after splenectomy: A systematic review and meta-analysis. Biomed Rep. 2016;5(4):483-490. doi: 10.3892/br.2016.755.

24. Wang H, Kopac D, Brisebois R, Sample C, Shapiro AM. Randomized controlled trial to investigate the impact of anticoagulation on the incidence of splenic or portal vein thrombosis after laparoscopic splenectomy. Can J Surg. 2011;54(4):227-31. doi: 10.1503/cjs.049909.

25. Qi X, Bai M, Guo X, Fan D. Pharmacologic prophylaxis of portal venous system thrombosis after splenectomy: a metaanalysis. Gastroenterol Res Pract. 2014;2014:292689. doi: $10.1155 / 2014 / 292689$.

26. Qi X. Portal Vein Thrombosis: Recent Advance. Adv Exp MedBiol.2017;906:229-39.doi:10.1007/5584_2016_118.

27. Congly SE, Lee SS. Portal vein thrombosis: should anticoagulation be used? Curr Gastroenterol Rep. 2013;15(2):306. doi: 10.1007/s11894-012-0306-0.

28. Amitrano L, Guardascione MA, Menchise A, Martino R, Scaglione M, Giovine S, et al. Safety and efficacy of anticoagulation therapy with low molecular weight heparin for portal vein thrombosis in patients with liver cirrhosis. J Clin Gastroenterol. 2010;44(6):448-51. doi: 10.1097/ MCG.0b013e3181b3ab44.

29. Smalberg JH, Spaander MV, Jie KS, Pattynama PM, van Buuren HR, van den Berg B, et al. Risks and benefits of transcatheter thrombolytic therapy in patients with splanchnic venous thrombosis. Thromb Haemost. 2008;100(6):1084-8.

30. Wang MQ, Guo LP, Lin HY, Liu FY, Duan F, Wang ZJ. Transradial approach for transcatheter selective superior mesenteric artery urokinase infusion therapy in patients with acute extensive portal and superior mesenteric vein thrombosis. Cardiovasc Intervent Radiol. 2010;33(1):80-9. doi: 10.1007/s00270-009-9777-2.

31. Wang MQ, Liu FY, Duan F, Wang ZJ, Song P, Fan QS. Acute symptomatic mesenteric venous thrombosis: treatment by catheter-directed thrombolysis with transjugular intrahepatic route. Abdom Imaging. 2011;36(4):390-8. doi: 10.1007/s00261-010-9637-1.

32. De Santis A, Moscatelli R, Catalano C, Iannetti A, Gigliotti F, Cristofari F, et al. Systemic thrombolysis of portal vein thrombosis in cirrhotic patients: a pilot study. Dig Liver Dis. 2010;42(6):451-5. doi: 10.1016/j.dld.2009.08.009.

33. Qi X, Han G. Transjugular intrahepatic portosystemic shunt in the treatment of portal vein thrombosis: a critical review of literature. Hepatol Int. 2012;6(3):576-90. doi: 10.1007/ s12072-011-9324-5.

34. Cao G, Ko GY, Sung KB, Yoon HK, Gwon DI, Kim JH. Treatment of postoperative main portal vein and superior mesenteric vein thrombosis with balloon angioplasty and/ or stent placement. Acta Radiol. 2013;54(5):526-32. doi: $10.1177 / 0284185113475917$. 
35. Crawford JM. Vascular disorders of the liver. Clin Liver Dis. 2010;14(4):635-50. doi: 10.1016/j.cld.2010.08.002.

36. Silva-Junior G, Turon F, Hernandez-Gea V, Darnell A, García-Criado Á, García-Pagán JC. Unexpected disappearance of portal cavernoma on long-term anticoagulation. J Hepatol. 2014;61(2):446-8. doi: 10.1016/j. jhep.2014.04.002.

37. Garcia-Tsao G, Abraldes JG, Berzigotti A, Bosch J. Portal hypertensive bleeding in cirrhosis: Risk stratification, diagnosis, and management: 2016 practice guidance by the American Association for the study of liver diseases. Hepatology. 2017;65(1):310-335. doi: 10.1002/hep.28906.

38. Abby Philips C, Sahney A. Oesophageal and gastric varices: historical aspects, classification and grading: everything in one place. Gastroenterol Rep (Oxf). 2016;4(3):186-95. doi: 10.1093/gastro/gow018.

39. Sarin SK, Kumar A. Endoscopic treatment of gastric varices. Clin Liver Dis. 2014;18(4):809-27. doi: 10.1016/j. cld.2014.07.004.

40. Lo GH. Endoscopic treatments for portal hypertension. Hepatol Int. 2018;12(Suppl 1):91-101. doi: 10.1007/ s12072-017-9828-8.

41. Sharma BC, Banka AK, Rawat A, Srivastava S. Gastric Varices in Cirrhosis versus Extrahepatic Portal Venous Obstruction and Response to Endoscopic N-Butyl-2cyanoacrylate Injection. J Clin Exp Hepatol. 2013;3(1):1923. doi: 10.1016/j.jceh.2013.01.002.

42. Qiao W, Ren Y, Bai Y, Liu S, Zhang Q, Zhi F. Cyanoacrylate Injection Versus Band Ligation in the Endoscopic Management of Acute Gastric Variceal Bleeding: Meta-Analysis of Randomized, Controlled Studies Based on the PRISMA Statement. Medicine (Baltimore). 2015;94(41):e1725. doi: 10.1097/ MD.0000000000001725.

43. Ríos Castellanos E, Seron P, Gisbert JP, Bonfill Cosp X. Endoscopic injection of cyanoacrylate glue versus other endoscopic procedures for acute bleeding gastric varices in people with portal hypertension. Cochrane Database Syst Rev. 2015;(5):CD010180. doi: 10.1002/14651858. CD010180.pub2.
44. Binmoeller KF, Weilert F, Shah JN, Kim J. EUS-guided transesophageal treatment of gastric fundal varices with combined coiling and cyanoacrylate glue injection (with videos). Gastrointest Endosc. 2011;74(5):1019-25. doi: 10.1016/j. gie.2011.06.030.

45. Sugimoto N, Watanabe K, Watanabe K, Ogata S, Shimoda R, Sakata $\mathrm{H}$, et al. Endoscopic hemostasis for bleeding gastric varices treated by combination of variceal ligation and sclerotherapy with N-butyl-2-cyanoacrylate. J Gastroenterol. 2007;42(7):528-32.doi: 10.1007/s00535-007-2041-0.

46. Mishra SR, Sharma BC, Kumar A, Sarin SK. Primary prophylaxis of gastric variceal bleeding comparing cyanoacrylate injection and beta-blockers: a randomized controlled trial. J Hepatol. 2011;54(6):1161-7. doi: 10.1016/j. jhep.2010.09.031.

47. McAvoy NC, Plevris JN, Hayes PC. Human thrombin for the treatment of gastric and ectopic varices. World J Gastroenterol. 2012;18(41):5912-7. doi: 10.3748/wjg.v18. i41.5912.

48. Min SK, Kim SG, Kim YS, Bae JY, Lee JC, Lee SH, et al. Comparison among endoscopic variceal obliteration, endoscopic band ligation, and balloon-occluded retrograde transvenous obliteration for treatment of gastric variceal bleeding. Korean J Gastroenterol. 2011 25;57(5):302-8.

49. Bai M, Qi XS, Yang ZP, Wu KC, Fan DM, Han GH. EVS vs TIPS shunt for gastric variceal bleeding in patients with cirrhosis: A meta-analysis. World J Gastrointest Pharmacol Ther. 2014;5(2):97-104. doi: 10.4292/wjgpt.v5.i2.97.

50. Shimamatsu K, Wanless IR. Role of ischemia in causing apoptosis, atrophy, and nodular hyperplasia in human liver. Hepatology. 1997;26(2):343-50. doi: 10.1002/ hep.510260214.

51. Marin D, Galluzzo A, Plessier A, Brancatelli G, Valla D, Vilgrain V. Focal nodular hyperplasia-like lesions in patients with cavernous transformation of the portal vein: prevalence, MR findings and natural history. Eur Radiol. 2011;21(10):2074-82. doi: 10.1007/s00330-011-2161-z.

52. Ibrahim M, Mostafa I, Devière J. New Developments in Managing Variceal Bleeding. Gastroenterology. 2018;154(7):1964-1969. doi: 10.1053/j.gastro.2018.02.023. 